\title{
A Gaussian bound for convolutions of functions on locally compact groups
}

by

\author{
Nick Dungey (Sydney)
}

\begin{abstract}
We give new and general sufficient conditions for a Gaussian upper bound on the convolutions $K_{m+n} * K_{m+n-1} * \cdots * K_{m+1}$ of a suitable sequence $K_{1}, K_{2}, K_{3}, \ldots$ of complex-valued functions on a unimodular, compactly generated locally compact group. As applications, we obtain Gaussian bounds for convolutions of suitable probability densities, and for convolutions of small perturbations of densities.
\end{abstract}

1. Introduction. Let $K_{1}, K_{2}, K_{3}, \ldots$ be a sequence of functions on a unimodular locally compact group $G$. The first main result of this paper gives very general sufficient conditions for a Gaussian upper bound on the convolved functions

$$
K_{m+n} * K_{m+n-1} * \cdots * K_{m+1}
$$

for all $m \in \mathbb{N}_{0}=\{0,1,2, \ldots\}$ and $n \in \mathbb{N}=\{1,2,3, \ldots\}$, where $*$ denotes convolution of functions on $G$. Examples where this result applies are discussed.

The problem of obtaining Gaussian estimates has been well studied in the important case of time homogeneous random walks, that is, in case $K_{n}=K$ is independent of $n$ and is a probability density on $G$. For example, see $[8,10,3]$ for results when $K=K_{n}$ is a symmetric density (symmetry means that $K(g)=K\left(g^{-1}\right)$ for all $\left.g \in G\right)$. More recently, Gaussian estimates for non-symmetric "centered" densities on groups of polynomial growth were obtained by Alexopoulos [1, 2]; see also [11, 6]. For relevant background material concerning random walks and diffusions on groups, see [12].

In the present paper, we move outside the random walk case and treat "time-inhomogeneous" convolutions of possibly complex-valued functions. This requires new effort since many of the previous methods for obtaining Gaussian estimates (for example, in $[8,1,2]$ ) do not obviously extend to the

2000 Mathematics Subject Classification: 60B15, 60G50, 22E30.

Key words and phrases: Gaussian bound, probability density, convolution, locally compact group, random walk. 
time-inhomogeneous case, nor to functions with negative or complex values. Our present method is based on some "convolution Nash inequalities" for unimodular groups recently introduced in $[4,5]$; in those papers, the inequalities were used to study the time-homogeneous case, that is, convolutions of a fixed complex-valued function $K$. We mention also the paper [7], where Gaussian estimates for complex heat kernels on Lie groups were obtained by quite different methods.

In Section 2 below we prove our main theorem giving Gaussian estimates, under some abstract assumptions including certain $L^{2}$ estimates for quadratic forms associated with $K_{n}$. As a byproduct of the proof, under weaker assumptions we obtain an $L^{\infty}$ estimate for convolutions.

In Section 3 we consider some examples where the main theorem can be applied. We first consider the case of probability densities, and show how to recover and extend previously known results of, for example, [8, 1, 2]. Then we obtain new results for functions which are suitable perturbations of probability densities.

2. The main theorems. In what follows, $G$ will be a unimodular locally compact group with Haar measure $d g$. We consider the spaces $L^{p}:=$ $L^{p}(G ; d g), 1 \leq p \leq \infty$, of complex-valued measurable functions, and use the notation $\|T\|_{p \rightarrow q}$ for the norm of a bounded linear operator $T: L^{p} \rightarrow L^{q}$.

Let $L=L_{G}$ be the left regular representation of $G$, so that $(L(g) f)(h)=$ $f\left(g^{-1} h\right), g, h \in G$, for a function $f: G \rightarrow \mathbb{C}$. Define also the difference operators $\partial_{g}:=L(g)-I, g \in G$, where $I$ denotes the identity operator on functions. For a locally integrable function $f: G \rightarrow \mathbb{C}$ we define the convolution operator $L(f)$ by setting

$$
\left(L(f) f_{1}\right)(g)=\left(f * f_{1}\right)(g)=\int_{G} d h f(h) f_{1}\left(h^{-1} g\right)
$$

for suitable functions $f_{1}: G \rightarrow \mathbb{C}$; this makes sense at least when $f_{1}$ is continuous and compactly supported, so that $L(f)$ has domain dense in $L^{p}$ for $1 \leq p<\infty$.

We shall assume that $G$ is compactly generated, and fix a relatively compact, open neighborhood $U$ of the identity $e$ of $G$ which is symmetric $(U=$ $\left.U^{-1}\right)$ and generates $G$. Thus $G=\bigcup_{n=1}^{\infty} U^{n}$ where $U^{n}:=\left\{g_{1} \cdots g_{n}: g_{1}, \ldots, g_{n}\right.$ $\in U\}$. Define the modulus $\varrho=\varrho_{U}: G \rightarrow \mathbb{N}$ by

$$
\varrho(g)=\inf \left\{n \in \mathbb{N}: g \in U^{n}\right\}, \quad g \in G .
$$

This has the basic properties that $\varrho(g)=\varrho\left(g^{-1}\right)$ and $\varrho(g h) \leq \varrho(g)+\varrho(h)$ for all $g, h \in G$. Let $V(r)$ be the $d g$-measure of the ball $B(r):=\{g \in G: \varrho(g)$ $\leq r\}$ for all $r \geq 1$. The multiplication operators $U_{\lambda}, \lambda \in \mathbb{R}$, are defined by

$$
U_{\lambda} f:=e^{\lambda \varrho} f
$$


for suitable $f: G \rightarrow \mathbb{C}$. For measurable $f: G \rightarrow \mathbb{C}$, we also define $\Gamma_{2}(f) \in$ $[0, \infty]$ by

$$
\Gamma_{2}(f)=\left(\int_{G} d g \int_{U} d u\left|\left(\partial_{u} f\right)(g)\right|^{2}\right)^{1 / 2}=\left(\int_{U} d u\left\|\partial_{u} f\right\|_{2}^{2}\right)^{1 / 2} .
$$

We will need the standard estimate (cf. [12, Proposition VII.3.2]) that

$$
\left\|\partial_{g} f\right\|_{2} \leq c_{U} \varrho(g) \Gamma_{2}(f) \quad \text { for all } g \in G \text { and } f \in L^{2},
$$

where $c_{U}$ is a constant depending only on $G, d g$ and $U$.

Let $\left(K_{n}\right)_{n \in \mathbb{N}}$ be a sequence of complex-valued measurable functions on $G$. Set

$$
T_{n} f:=K_{n} * f=L\left(K_{n}\right) f, \quad T_{n, \lambda} f:=U_{\lambda} T_{n} U_{-\lambda} f,
$$

and define associated quadratic forms $Q_{n, \lambda}$ by

$$
Q_{n, \lambda}(f):=\|f\|_{2}^{2}-\left\|T_{n, \lambda} f\right\|_{2}^{2} \quad \text { for } n \in \mathbb{N}, \lambda \in \mathbb{R} \text { and } f \in L^{2} .
$$

We can now state our main theorem.

TheOREm 2.1. Suppose that the functions $\left(K_{n}\right)_{n \in \mathbb{N}}$ satisfy the following Assumptions (I)-(III).

(I) There exist $c, b>0$ such that

$$
\left|K_{n}(g)\right| \leq c e^{-b \varrho(g)^{2}} \quad \text { for all } n \in \mathbb{N} \text { and } g \in G .
$$

(II) There are $c, \omega>0$ such that

$$
Q_{n, \lambda}(f) \geq c^{-1} \Gamma_{2}(f)^{2}-\omega \lambda^{2}\|f\|_{2}^{2} \quad \text { for all } n \in \mathbb{N},|\lambda| \leq 1 \text { and } f \in L^{2} .
$$

(III) There exist $a, D>0$ such that $V(r) \geq a r^{D}$ for all $r \geq 1$.

Then there are constants $c^{\prime}, b^{\prime}>0$, depending only on $G, d g, U$ and on the constants $c, b, \omega, a, D$ in Assumptions (I)-(III), such that

$$
\left|\left(K_{m+n} * K_{m+n-1} * \cdots * K_{m+1}\right)(g)\right| \leq c^{\prime} n^{-D / 2} e^{-b^{\prime} \varrho(g)^{2} / n}
$$

for all $m \in \mathbb{N}_{0}=\{0,1,2, \ldots\}, n \in \mathbb{N}$ and $g \in G$.

We remark that Assumption (II) of Theorem 2.1 implies that the operators $T_{n}=L\left(K_{n}\right)$ are contractions in $L^{2}$. But for complex-valued $K_{n}$, the $T_{n}$ need not be contractions in $L^{1}$ (or in $L^{\infty}$ ), which is the main obstruction to applying standard arguments for densities (for example, see [8, Section 2]) in the complex case.

Theorem 2.1 should be compared with [4, Theorem 2.3] where Gaussian estimates for convolution powers of a fixed complex-valued function $K$ were obtained. The hypotheses in [4] are different, however, and do not seem to adapt easily to the time-inhomogeneous case.

We proceed with the proof of Theorem 2.1, which requires several lemmas. 
LEMma 2.2. There exists $k>0$ such that

$$
\left\|T_{n, \lambda}\right\|_{2 \rightarrow 2} \leq e^{k \lambda^{2}} \quad \text { for all } n \in \mathbb{N}, \lambda \in \mathbb{R} .
$$

Therefore,

$$
\left\|T_{m+n, \lambda} \cdots T_{m+1, \lambda}\right\|_{2 \rightarrow 2} \leq e^{k \lambda^{2} n} \quad \text { for all } m \in \mathbb{N}_{0}, n \in \mathbb{N} \text { and } \lambda \in \mathbb{R}
$$

Proof. The second statement of the lemma follows immediately from the first statement. To prove the first statement, we observe from Assumption (II) of Theorem 2.1 that

$$
\|f\|_{2}^{2}-\left\|T_{n, \lambda} f\right\|_{2}^{2} \geq-\omega \lambda^{2}\|f\|_{2}^{2} \quad \text { for all } f \in L^{2} \text { and }|\lambda| \leq 1 .
$$

Therefore, $\left\|T_{n, \lambda} f\right\|_{2}^{2} \leq\left(1+\omega \lambda^{2}\right)\|f\|_{2}^{2}$ and

$$
\left\|T_{n, \lambda}\right\|_{2 \rightarrow 2} \leq\left(1+\omega \lambda^{2}\right)^{1 / 2} \leq 1+\omega \lambda^{2} \leq e^{\omega \lambda^{2}} \quad \text { whenever }|\lambda| \leq 1 .
$$

When $|\lambda| \geq 1$, a similar bound follows by an elementary integration of the Gaussian bound of Assumption (I); we omit the standard details (one uses the fact that $\int_{G} d g e^{-\delta \varrho(g)^{2}}$ is finite for any $\left.\delta>0\right)$.

LEMMA 2.3. There exist $c, k>0$ such that

$$
\left\|U_{\lambda} K_{n}\right\|_{2} \leq c e^{k \lambda^{2}} \quad \text { for all } n \in \mathbb{N} \text { and } \lambda \geq 0 \text {. }
$$

Proof. This also follows by integration of the bound of Assumption (I).

In the next lemma, which gives a "weighted convolution Nash inequality", we assume that $w: G \rightarrow(0, \infty)$ is any Borel measurable function such that $w(g)=w\left(g^{-1}\right), g \in G$, and such that $w, 1 / w$ are locally bounded. For convenience, we also denote by $w$ the operator of pointwise multiplication $f \mapsto w f$.

LEMMA $2.4([4])$. Let $w$ be as above. For $r>0$ write $\|w\|_{\infty, r}=\sup \{w(g)$ : $g \in G, \varrho(g) \leq r\}$. If $f$ is a locally integrable function on $G$ and $w f \in L^{2}$, then

$$
\|w f\|_{2} \leq \sup _{g \in G, \varrho(g) \leq r}\left\|w \partial_{g} f\right\|_{2}+\|w\|_{\infty, r} V(r)^{-1 / 2}\left\|w^{-1} L(f) w\right\|_{2 \rightarrow 2}
$$

for all $r>0$ (here, the right side is permitted to be infinite).

Proof. This is contained in [4, Lemma 2.2].

Proposition 2.5. There exist constants $c, k>1$ such that

$$
\left\|U_{\lambda} f\right\|_{2}^{2} \leq c r^{2}\left\{Q_{n, \lambda}\left(U_{\lambda} f\right)+k \lambda^{2}\left\|U_{\lambda} f\right\|_{2}^{2}\right\}+c r^{-D}\left(\left\|U_{-\lambda} L(f) U_{\lambda}\right\|_{2 \rightarrow 2}\right)^{2}
$$

for all $r \geq 1, \lambda \geq 0, n \in \mathbb{N}$ and locally integrable $f$ such that $U_{\lambda} f \in L^{2}$.

Proof. Since $c k>1$, the desired inequality holds trivially whenever $\lambda r \geq 1$. Thus, in the rest of the proof we may assume that $\lambda \geq 0$ and 
$r \geq 1$ satisfy $\lambda r \leq 1$; note that then $\lambda \leq 1$. Setting $w=e^{\lambda \varrho}$, squaring the inequality of Lemma 2.4 and applying Assumption (III) gives

$$
\left\|U_{\lambda} f\right\|_{2}^{2} \leq c \sup _{g \in G, \varrho(g) \leq r}\left\|U_{\lambda} \partial_{g} f\right\|_{2}^{2}+c r^{-D}\left(\left\|U_{-\lambda} L(f) U_{\lambda}\right\|_{2 \rightarrow 2}\right)^{2}
$$

whenever $r \geq 1$ and $\lambda r \leq 1$. Therefore, to complete the proof it suffices to establish an inequality of the form

$$
\left\|U_{\lambda} \partial_{g} f\right\|_{2}^{2} \leq c \varrho(g)^{2}\left\{Q_{n, \lambda}\left(U_{\lambda} f\right)+k \lambda^{2} e^{c \varrho(g) \lambda}\left\|U_{\lambda} f\right\|_{2}^{2}\right\}
$$

for all $g \in G, 0 \leq \lambda \leq 1$ and $n \in \mathbb{N}$. To do this, observe the identity

$$
U_{\lambda} \partial_{g} f=\partial_{g} U_{\lambda} f-\left[1-e^{-\lambda \partial_{g} \varrho}\right] L(g) U_{\lambda} f .
$$

From (1) and Assumption (II),

$$
\left\|\partial_{g} U_{\lambda} f\right\|_{2}^{2} \leq c \varrho(g)^{2} \Gamma_{2}\left(U_{\lambda} f\right)^{2} \leq c^{\prime} \varrho(g)^{2}\left\{Q_{n, \lambda}\left(U_{\lambda} f\right)+c^{\prime \prime} \lambda^{2}\left\|U_{\lambda} f\right\|_{2}^{2}\right\}
$$

for all $0 \leq \lambda \leq 1$. Also, since $\left|1-e^{s}\right| \leq|s| e^{|s|}$ for $s \in \mathbb{R}$, and $\left\|\partial_{g} \varrho\right\|_{\infty} \leq \varrho(g)$, we find that

$$
\left\|\left[1-e^{-\lambda \partial_{g} \varrho}\right] L(g) U_{\lambda} f\right\|_{2} \leq\left\|1-e^{-\lambda \partial_{g} \varrho}\right\|_{\infty}\left\|U_{\lambda} f\right\|_{2} \leq \lambda \varrho(g) e^{\lambda \varrho(g)}\left\|U_{\lambda} f\right\|_{2}
$$

for all $\lambda \geq 0$. Then (2) follows.

To continue the proof of Theorem 2.1, let us fix $k>1$ large enough so that the estimates of Lemmas 2.2, 2.3 and Proposition 2.5 hold for this $k$. Put

$$
P_{m, n}:=K_{m+n} * \cdots * K_{m+1}, \quad J_{m, \lambda}(n):=e^{-2 k \lambda^{2} n}\left\|U_{\lambda} P_{m, n}\right\|_{2}^{2}
$$

for all $m \in \mathbb{N}_{0}, n \in \mathbb{N}$ and $\lambda \geq 0$. We claim that the function $n \mapsto J_{m, \lambda}(n)$ is non-increasing in $n$; indeed, since

$$
U_{\lambda} P_{m, n+1}=U_{\lambda}\left(K_{m+n+1} * P_{m, n}\right)=T_{m+n+1, \lambda}\left(U_{\lambda} P_{m, n}\right)
$$

we know by Lemma 2.2 that

$$
\begin{aligned}
J_{m, \lambda}(n+1) & =e^{-2 k \lambda^{2}(n+1)}\left\|T_{m+n+1, \lambda}\left(U_{\lambda} P_{m, n}\right)\right\|_{2}^{2} \\
& \leq e^{-2 k \lambda^{2} n}\left\|U_{\lambda} P_{m, n}\right\|_{2}^{2}=J_{m, \lambda}(n)
\end{aligned}
$$

for all $m \in \mathbb{N}_{0}, n \in \mathbb{N}$ and $\lambda \geq 0$. Moreover, Lemma 2.3 implies that $\sup \left\{J_{m, \lambda}(1): m \in \mathbb{N}_{0}, \lambda \geq 0\right\} \leq c_{0}$ for some constant $c_{0}>0$. Hence

$$
\sup \left\{J_{m, \lambda}(n): m \in \mathbb{N}_{0}, n \in \mathbb{N}, \lambda \geq 0\right\} \leq c_{0},
$$

since $J_{m, \lambda}(n)$ is non-increasing in $n$.

Next, suppose $0 \leq \lambda \leq 1$ and in Proposition 2.5 set

$$
f=e^{-k \lambda^{2} n} P_{m, n} .
$$

Observe that $\left\|U_{\lambda} f\right\|_{2}^{2}=J_{m, \lambda}(n)$, that

$$
\begin{aligned}
\left\|U_{-\lambda} L(f) U_{\lambda}\right\|_{2 \rightarrow 2} & =e^{-k \lambda^{2} n}\left\|U_{-\lambda} T_{m+n} \cdots T_{m+1} U_{\lambda}\right\|_{2 \rightarrow 2} \\
& =e^{-k \lambda^{2} n}\left\|T_{m+n,-\lambda} \cdots T_{m+1,-\lambda}\right\|_{2 \rightarrow 2} \leq 1
\end{aligned}
$$


by Lemma 2.2 , and that

$$
\begin{aligned}
& Q_{m+n+1, \lambda}\left(U_{\lambda} f\right)+k \lambda^{2}\left\|U_{\lambda} f\right\|_{2}^{2} \\
& \quad=e^{-2 k \lambda^{2} n}\left\|U_{\lambda} P_{m, n}\right\|_{2}^{2}-e^{-2 k \lambda^{2} n}\left\|U_{\lambda} P_{m, n+1}\right\|_{2}^{2}+k \lambda^{2} e^{-2 k \lambda^{2} n}\left\|U_{\lambda} P_{m, n}\right\|_{2}^{2} \\
& \quad \leq e^{2 k \lambda^{2}}\left[e^{-2 k \lambda^{2} n}\left\|U_{\lambda} P_{m, n}\right\|_{2}^{2}-e^{-2 k \lambda^{2}(n+1)}\left\|U_{\lambda} P_{m, n+1}\right\|_{2}^{2}\right] \\
& \quad \leq e^{2 k}\left[J_{m, \lambda}(n)-J_{m, \lambda}(n+1)\right]
\end{aligned}
$$

where the last step used that $\lambda \leq 1$. Thus Proposition 2.5 shows for some $c>0$ that

$$
J_{m, \lambda}(n) \leq c r^{2}\left[J_{m, \lambda}(n)-J_{m, \lambda}(n+1)\right]+c r^{-D},
$$

which implies, since $J_{m, \lambda}(n+1) \leq J_{m, \lambda}(n)$, that

$$
J_{m, \lambda}(n+1) \leq c r^{2}\left[J_{m, \lambda}(n)-J_{m, \lambda}(n+1)\right]+c r^{-D}
$$

for all $r \geq 1,0 \leq \lambda \leq 1, m \in \mathbb{N}_{0}$ and $n \in \mathbb{N}$. In this inequality, we may assume that $c$ is large enough that $2 c \geq c_{0}$ where $c_{0}$ is as in (3). Then choosing $r$ in (4) so that $c r^{-D}=2^{-1} J_{m, \lambda}(n+1)$, we have $r \geq 1$, and (4) yields an estimate

$$
J_{m, \lambda}(n+1)^{1+(2 / D)} \leq c^{\prime}\left[J_{m, \lambda}(n)-J_{m, \lambda}(n+1)\right]
$$

for all $m \in \mathbb{N}_{0}, n \in \mathbb{N}$ and $0 \leq \lambda \leq 1$.

The following lemma now allows us to estimate $J_{m, \lambda}(n)$. The lemma is not new (see for example [12, Lemma VI.3.5]), but we give a proof for the sake of completeness.

Lemma 2.6. Let $c_{0}, c_{1}, D>0$, and let $(\gamma(n))_{n \in \mathbb{N}}$ be a sequence of nonnegative real numbers satisfying $\gamma(1) \leq c_{0}$ and

$$
\gamma(n+1)^{1+(2 / D)} \leq c_{1}(\gamma(n)-\gamma(n+1)) \quad \text { for all } n \in \mathbb{N} .
$$

Then there exists a constant $c_{2}>0$, depending only on $c_{0}, c_{1}, D$, such that

$$
\gamma(n) \leq c_{2} n^{-D / 2} \quad \text { for all } n \in \mathbb{N} .
$$

Proof. Let $c_{2}$ be large enough that $c_{2}>c_{0}$; we will choose $c_{2}$ more precisely later. If the desired estimate of the lemma did not hold, then for some $n \in \mathbb{N}$ we must have

$$
\gamma(n) \leq c_{2} n^{-D / 2}, \quad \gamma(n+1)>c_{2}(n+1)^{-D / 2} .
$$

We would then have

$$
\gamma(n)-\gamma(n+1) \leq c_{2}\left(n^{-D / 2}-(n+1)^{-D / 2}\right) \leq c_{2} c(D)(n+1)^{-(D / 2)-1}
$$

where $c(D)>0$ is a constant depending only on $D$. The hypothesis then implies that

$$
\gamma(n+1)^{1+(2 / D)} \leq c_{1} c_{2} c(D)(n+1)^{-(D / 2)-1}
$$


or equivalently,

$$
\gamma(n+1) \leq\left(c_{1} c_{2} c(D)\right)^{D /(D+2)}(n+1)^{-D / 2} .
$$

Now fixing $c_{2}>c_{0}$ large enough so that

$$
\left(c_{1} c_{2} c(D)\right)^{D /(D+2)}<c_{2},
$$

we see that the last estimate contradicts (6). The lemma follows.

From (3), (5) and Lemma 2.6 we infer an estimate

or, in other words,

$$
J_{m, \lambda}(n) \leq c n^{-D / 2}
$$

$$
\left\|U_{\lambda} P_{m, n}\right\|_{2} \leq c^{\prime} n^{-D / 4} e^{k \lambda^{2} n}
$$

for all $m \in \mathbb{N}_{0}, n \in \mathbb{N}$ and $0 \leq \lambda \leq 1$. When $\lambda \geq 1$, it follows from (3) that

$$
\left\|U_{\lambda} P_{m, n}\right\|_{2} \leq c_{0}^{1 / 2} e^{k \lambda^{2} n} \leq c_{0}^{1 / 2} e^{-n} e^{(k+1) \lambda^{2} n} .
$$

We conclude that for some $k>0$, an estimate of form (7) holds for all $m \in \mathbb{N}_{0}, n \in \mathbb{N}$ and $\lambda \geq 0$.

The desired Gaussian bound of Theorem 2.1 is a standard consequence of (7). In fact, from the convolution identity

$$
P_{m, n}=P_{m+n_{1}, n_{2}} * P_{m, n_{1}}
$$

where $n=n_{1}+n_{2}$, we easily deduce a bound

$$
\begin{aligned}
e^{\lambda \varrho(g)}\left|P_{m, n}(g)\right| & \leq \int_{G} d h e^{\lambda \varrho(h)}\left|P_{m+n_{1}, n_{2}}(h)\right| e^{\lambda \varrho\left(h^{-1} g\right)}\left|P_{m, n_{1}}\left(h^{-1} g\right)\right| \\
& \leq\left\|U_{\lambda} P_{m+n_{1}, n_{2}}\right\|_{2}\left\|U_{\lambda} P_{m, n_{1}}\right\|_{2} \leq c n^{-D / 2} e^{k \lambda^{2} n}
\end{aligned}
$$

for all $g \in G, m \in \mathbb{N}_{0}, n \in\{2,3,4, \ldots\}$ and $\lambda \geq 0$. Choosing $\lambda$ to be a small constant multiple of $\varrho(g) / n$ ends the proof of Theorem 2.1.

The following corollary of Theorem 2.1 gives an alternative form of Assumption (II) which is often more convenient in practice (for examples, see Section 3).

Corollary 2.7. Suppose $\left(K_{n}\right)_{n \in \mathbb{N}}$ satisfy Assumptions (I) and (III) of Theorem 2.1 and the following assumption.

(II)' There exists $c \geq 1$ with

$$
Q_{n, 0}(f) \geq c^{-1} \Gamma_{2}(f)^{2},
$$

and there exists $\varepsilon_{0}>0$ such that

$$
\left|Q_{n, \lambda}(f)-Q_{n, 0}(f)\right| \leq \varepsilon \Gamma_{2}(f)^{2}+c(\varepsilon) \lambda^{2}\|f\|_{2}^{2}
$$

for all $\varepsilon \in\left(0, \varepsilon_{0}\right],|\lambda| \leq 1$ and $f \in L^{2}$, where $c(\varepsilon)>0$ may depend on $\varepsilon$.

Then Assumption (II) of Theorem 2.1 holds, and hence the conclusion of Theorem 2.1 holds. 
Proof. Assumption (II)' implies that

$$
\left|Q_{n, \lambda}(f)-Q_{n, 0}(f)\right| \leq c \varepsilon Q_{n, 0}(f)+c(\varepsilon) \lambda^{2}\|f\|_{2}^{2}
$$

for all $|\lambda| \leq 1$ and $f \in L^{2}$. Hence, fixing an $\varepsilon \in\left(0, \varepsilon_{0}\right]$ small enough that $c \varepsilon \leq 2^{-1}$, we have

$$
\begin{aligned}
Q_{n, \lambda}(f) & \geq Q_{n, 0}(f)-\left|Q_{n, \lambda}(f)-Q_{n, 0}(f)\right| \\
& \geq 2^{-1} Q_{n, 0}(f)-c^{\prime} \lambda^{2}\|f\|_{2}^{2} \geq 2^{-1} c^{-1} \Gamma_{2}(f)^{2}-c^{\prime} \lambda^{2}\|f\|_{2}^{2},
\end{aligned}
$$

which establishes Assumption (II).

To conclude this section, we describe a uniform $L^{\infty}$ estimate for convolutions under weaker hypotheses than Theorem 2.1. This result is related to estimates of [5] for convolution powers of a fixed complex-valued function $K$; it also generalizes well known results for probability densities (see [12, p. 88] and references therein).

THEOREM 2.8. Let $\left(K_{n}\right)_{n \in \mathbb{N}}$ be a sequence of complex-valued measurable functions on $G$ satisfying the following assumptions.

(I) $\left\|K_{n}\right\|_{2} \leq c$ for all $n \in \mathbb{N}$.

(II) $Q_{n, 0}(f) \geq c^{-1} \Gamma_{2}(f)^{2}$ for all $n \in \mathbb{N}$ and $f \in L^{2}$.

(III) There exist $a, D>0$ such that $V(r) \geq a r^{D}$ for all $r \geq 1$.

Then there exist $c^{\prime}, c^{\prime \prime}>0$ (depending only on $\left.G, d g, U, c, a, D\right)$ such that

$$
\left\|K_{m+n} * \cdots * K_{m+1}\right\|_{2} \leq c^{\prime} n^{-D / 4} \quad \text { for all } m \in \mathbb{N}_{0}, n \in \mathbb{N},
$$

and

$$
\left\|K_{m+n} * \cdots * K_{m+1}\right\|_{\infty} \leq c^{\prime \prime} n^{-D / 2} \quad \text { for all } m \in \mathbb{N}_{0}, n \in\{2,3,4, \ldots\} .
$$

Proof. This is just a simpler version of the proof of Theorem 2.1, and we shall leave details to the reader. Observe that the estimates of Lemma 2.2, Lemma 2.3 and Proposition 2.5 go through in case $\lambda=0$. Then one may derive the following difference inequality for the quantity $J_{m}(n):=\left\|P_{m, n}\right\|_{2}^{2}$ :

$$
J_{m}(n+1)^{1+(2 / D)} \leq c\left[J_{m}(n)-J_{m}(n+1)\right]
$$

for all $m \in \mathbb{N}_{0}$ and $n \in \mathbb{N}$. Thus Lemma 2.6 can be applied.

3. Examples. In this section, we continue the notations of Section 2 and describe examples where Theorems 2.1 or 2.8 apply.

Let $G$ be a unimodular, compactly generated locally compact group. Throughout the section we shall assume that $G$ satisfies the volume bound $V(r) \geq a r^{D}, r \geq 1$, for some $D>0$. Given $F: G \rightarrow \mathbb{C}$ we define $\widetilde{F}(g):=$ $F\left(g^{-1}\right)$ for $g \in G$, and say that $F$ is symmetric in case $F=\widetilde{F}$.

ExAmple (i). Let $\left(K_{n}\right)_{n \in \mathbb{N}}$ be probability densities on $G$, that is, $0 \leq$ $K_{n} \in L^{1}$ and $\int_{G} K_{n}=1$. Suppose also that $K_{n}$ is symmetric, that Assump- 
tion (I) of Theorem 2.1 holds, and finally that

$$
\inf \left\{K_{n}(g): g \in U^{\prime}, n \in \mathbb{N}\right\}>0
$$

where $U^{\prime} \subseteq G$ is some relatively compact, open, symmetric $\left(U^{\prime}=\left(U^{\prime}\right)^{-1}\right)$ generating neighborhood of $e$.

We claim that Assumption (II) ${ }^{\prime}$ of Corollary 2.7 holds, and hence the Gaussian estimate of Theorem 2.1 applies. To verify Assumption (II)' requires some work. We begin with

Lemma 3.1. One has

$$
f-F * f=2^{-1} \int_{G} d g F(g) \partial_{g^{-1}} \partial_{g} f
$$

for all $f \in L^{2}$ and all symmetric $F \in L^{1}$ satisfying $\int_{G} F=1$. Also,

$$
\|f\|_{2}^{2}-\|F * f\|_{2}^{2}=2^{-1} \int_{G} d g(\widetilde{F} * F)(g)\left\|\partial_{g} f\right\|_{2}^{2}
$$

for all $f \in L^{2}$ and all real-valued $F \in L^{1}$ satisfying $\int_{G} F=1$.

Proof. The first statement of the lemma is obtained by a straightforward calculation. To prove the second statement, observe that the symmetric function $F^{\prime}:=\widetilde{F} * F$ satisfies $\int_{G} F^{\prime}=1$ and

$$
\|f\|_{2}^{2}-\|F * f\|_{2}^{2}=\left(f-F^{\prime} * f, f\right)=2^{-1} \int_{G} d g F^{\prime}(g)\left(\partial_{g^{-1}} \partial_{g} f, f\right) .
$$

The lemma follows.

From the second part of Lemma 3.1 and (9), we deduce an inequality

$$
Q_{n, 0}(f)=\|f\|_{2}^{2}-\left\|K_{n} * f\right\|_{2}^{2} \geq c^{-1} \int_{U^{\prime}} d u\left\|\partial_{u} f\right\|_{2}^{2} .
$$

It follows that $Q_{n, 0}(f) \geq\left(c^{\prime}\right)^{-1} \Gamma_{2}(f)^{2}$, by a version of (1) with the neighborhood $U^{\prime}$ replacing $U$.

To verify Assumption (II) ${ }^{\prime}$, it remains to prove an estimate (8). To do this, first consider the quadratic forms $R_{n, \lambda}$ defined by

$$
R_{n, \lambda}(f):=\left(\left(I-T_{n, \lambda}\right) f, f\right) \quad \text { for } n \in \mathbb{N} \text { and } \lambda \in \mathbb{R} .
$$

The next lemma uses standard ideas and could be extracted, for example, from $[8,6]$, but for the sake of completeness we sketch a proof.

Lemma 3.2. For each $\varepsilon>0$ there exists $c(\varepsilon)>0$ such that

$$
\left|R_{n, \lambda}(f)-R_{n, 0}(f)\right| \leq \varepsilon \Gamma_{2}(f)^{2}+c(\varepsilon) \lambda^{2}\|f\|_{2}^{2}
$$

for all $n \in \mathbb{N},|\lambda| \leq 1$ and $f \in L^{2}$.

Proof. By Lemma 3.1 and symmetry of $K_{n}$, we find that

$$
R_{n, \lambda}(f)-R_{n, 0}(f)=2^{-1} \int_{G} d g K_{n}(g)\left(\left[U_{\lambda} \partial_{g^{-1}} \partial_{g} U_{-\lambda}-\partial_{g^{-1}} \partial_{g}\right] f, f\right) .
$$


But applying the identity $U_{\lambda} \partial_{g} U_{-\lambda}=\partial_{g}-\left[1-e^{-\lambda \partial_{g} \varrho}\right] L(g)$ and using (1) and $\left\|1-e^{-\lambda \partial_{g} \varrho}\right\|_{\infty} \leq|\lambda| \varrho(g) e^{|\lambda| \varrho(g)}$ (compare the proof of Proposition 2.5), one may show that

$$
\begin{aligned}
\left|\left(\left[U_{\lambda} \partial_{g} \partial_{h} U_{-\lambda}-\partial_{g} \partial_{h}\right] f, f\right)\right| & =\left|\left(U_{\lambda} \partial_{h} U_{-\lambda} f, U_{-\lambda} \partial_{g^{-1}} U_{\lambda} f\right)-\left(\partial_{h} f, \partial_{g^{-1}} f\right)\right| \\
& \leq c e^{c(\varrho(g)+\varrho(h))}\left(|\lambda|^{2}\|f\|_{2}^{2}+|\lambda|\|f\|_{2} \Gamma_{2}(f)\right)
\end{aligned}
$$

for all $|\lambda| \leq 1, g, h \in G$ and $f \in L^{2}$. The lemma follows easily.

We can relate $R_{n, \lambda}$ and $Q_{n, \lambda}$ by observing that

$$
\begin{aligned}
Q_{n, 0}(f)-Q_{n, \lambda}(f)= & \left\|T_{n, \lambda} f\right\|_{2}^{2}-\left\|T_{n} f\right\|_{2}^{2} \\
= & \left(T_{n} f,\left(T_{n, \lambda}-T_{n}\right) f\right)+\left(\left(T_{n, \lambda}-T_{n}\right) f, T_{n} f\right) \\
& +\left(\left(T_{n, \lambda}-T_{n}\right) f,\left(T_{n, \lambda}-T_{n}\right) f\right) \\
= & 2 \operatorname{Re}\left(\left(T_{n, \lambda}-T_{n}\right) f, T_{n} f\right)+\left\|\left(T_{n, \lambda}-T_{n}\right) f\right\|_{2}^{2} \\
= & 2 \operatorname{Re}\left\{R_{n, 0}(f)-R_{n, \lambda}(f)\right\}+\left\|\left(T_{n, \lambda}-T_{n}\right) f\right\|_{2}^{2} \\
& -2 \operatorname{Re}\left(\left(T_{n, \lambda}-T_{n}\right) f,\left(I-T_{n}\right) f\right) .
\end{aligned}
$$

Then (8) follows from Lemma 3.2 and from the estimates

$$
\left\|\left(I-T_{n}\right) f\right\|_{2} \leq c \Gamma_{2}(f), \quad\left\|\left(T_{n}-T_{n, \lambda}\right) f\right\|_{2} \leq c|\lambda|\|f\|_{2}
$$

for all $n \in \mathbb{N}, f \in L^{2}$ and $|\lambda| \leq 1$. Estimates (10) are easily proved even for non-symmetric densities: first observe that

$$
\left(I-T_{n}\right) f=\int_{G} d g K_{n}(g)(f-L(g) f)=-\int_{G} d g K_{n}(g) \partial_{g} f
$$

and apply (1) and Assumption (I), and secondly, write

$$
\begin{aligned}
\left(T_{n}-T_{n, \lambda}\right) f & =\int_{G} d g K_{n}(g)\left(L(g)-U_{\lambda} L(g) U_{-\lambda}\right) f \\
& =\int_{G} d g K_{n}(g)\left[1-e^{-\lambda \partial_{g} \varrho}\right] L(g) f
\end{aligned}
$$

in order to deduce the second estimate of (10).

Example (ii). To generalize Example (i), let $\left(K_{n}\right)_{n \in \mathbb{N}}$ be compactly supported densities on $G$ which are not necessarily symmetric, but which are centered in the following sense.

Consider the projection $\pi_{0}: G \rightarrow G / G_{0}$, where $G_{0}:=\overline{[G, G]}$ is the closure in $G$ of the commutator subgroup $[G, G]$. Now $G / G_{0}$ is a compactly generated locally compact abelian group, so by a standard theorem $([9$, Theorem II.9.8]) it is isomorphic with $\mathbb{R}^{v} \times \mathbb{Z}^{w} \times A$ where $v, w \in \mathbb{N}_{0}$ and $A$ is a compact abelian group. One says that $K_{n}$ is centered if

$$
\int_{G} d g K_{n}(g) \pi_{0}^{(j)}(g)=0
$$


for all $j \in\{1, \ldots, v+w\}$, where $\pi_{0}^{(j)}: G \rightarrow \mathbb{R}$ are the components in $\mathbb{R}^{v} \times \mathbb{Z}^{w}$ of the mapping $\pi_{0}: G \rightarrow \mathbb{R}^{v} \times \mathbb{Z}^{w} \times A$. (This definition generalizes the special cases where $G$ is a Lie group or a discrete group, studied in $[1,2,11,6]$.)

Assume that the $K_{n}$ are centered and have uniform compact support in the sense that $K_{n} \leq c \chi_{W}$ for all $n \in \mathbb{N}$ and some fixed compact set $W \subseteq G$. Suppose moreover that (9) holds.

We claim that Assumption (II) ${ }^{\prime}$ of Corollary 2.7 holds, and hence the Gaussian estimate of Theorem 2.1 applies. This Gaussian estimate extends results of Alexopoulos [1,2] to time-inhomogeneous random walks. Note that the methods of $[1,2]$ are completely different, being based on homogenization theory, and do not appear to work in the time-inhomogeneous case.

The proof of Assumption (II)' follows the arguments of Example (i), with the exception that the proof of the estimate of Lemma 3.2 is now more difficult. See [6] (especially Section 4) for proof of this estimate in the case where $G$ is discrete; the general case requires more effort and the details will appear elsewhere. The essential idea is that if $K_{n}$ is centered then $I-T_{n}$ is a second-order difference operator with no first-order terms; more precisely, $I-T_{n}$ is expressible as a linear combination of monomial difference operators, with each monomial having the form

$$
\partial_{g_{1}} \ldots \partial_{g_{s}}
$$

for some $s \geq 2$ and $g_{1}, \ldots, g_{s} \in G$ (for a proof of this assertion when $G$ is discrete, see [6]). Given this result, one may reason as in the proof of Lemma 3.2. We omit further details.

ExAmple (iii). This is an example for Theorem 2.8. Let $\left(K_{n}\right)_{n \in \mathbb{N}}$ be densities satisfying $\left\|K_{n}\right\|_{2} \leq c$ for all $n$ and satisfying (9); no assumption of symmetry or centeredness is required. Then Assumption (II) of Theorem 2.8 holds (this follows from (9) and Lemma 3.1), so that the conclusion of Theorem 2.8 applies.

The result of Example (iii) was proved by different methods in [12, Chapter VII].

In the remaining two examples, we derive apparently new estimates for convolutions of perturbations of densities. Example (iv) extends the uniform estimate of Example (iii) to perturbations, while Example (v) extends the Gaussian estimate of Example (i).

EXAMPLE (iv). Let $\left(K_{n}\right)_{n \in \mathbb{N}} \subseteq L^{1} \cap L^{2}$ be densities satisfying the same assumptions as in Example (iii). Suppose $\left(M_{n}\right)_{n \in \mathbb{N}}$ are real-valued functions on $G$ with $M_{n} \in L^{1} \cap L^{2}, \int_{G} M_{n}=0$, and $\left\|M_{n}\right\|_{2} \leq c$ for all $n \in \mathbb{N}$. Suppose also that

$$
\int_{G} d g K_{n}(g) \varrho(g)^{2}+\int_{G} d g\left|M_{n}(g)\right| \varrho(g)^{2} \leq c \quad \text { for all } n \in \mathbb{N} .
$$


Define perturbations of the $K_{n}$ by setting

$$
K_{n}^{\tau}:=K_{n}+\tau M_{n} \quad \text { for all } \tau \in \mathbb{R} .
$$

The $K_{n}^{\tau}$ might take negative values, so are not densities in general. We have

THEOREM 3.3. There is an $s_{0}>0$ such that

$$
\left\|K_{m+n}^{\tau_{n}} * \cdots * K_{m+1}^{\tau_{1}}\right\|_{\infty} \leq c n^{-D / 2}
$$

for all $m \in \mathbb{N}_{0}, n \in\{2,3,4, \ldots\}$ and $\tau_{1}, \ldots, \tau_{n} \in \mathbb{R}$ satisfying $\left|\tau_{j}\right|<s_{0}$ for all $j$.

Proof. First note that $\left\|K_{n}^{\tau}\right\|_{2} \leq c^{\prime}$ for all $n \in \mathbb{N}$ and all $\tau \in \mathbb{R}$ with $|\tau| \leq 1$. Next, since $\int_{G} K_{n}^{\tau}=1$ it follows from the second statement of Lemma 3.1 that

$$
\begin{aligned}
\|f\|_{2}^{2}-\left\|K_{n}^{\tau} * f\right\|_{2}^{2}= & 2^{-1} \int_{G} d g\left(\widetilde{K}_{n} * K_{n}\right)(g)\left\|\partial_{g} f\right\|_{2}^{2} \\
& +2^{-1} \tau \int_{G} d g\left(\left(\widetilde{K}_{n} * M_{n}\right)(g)+\left(\widetilde{M}_{n} * K_{n}\right)(g)\right)\left\|\partial_{g} f\right\|_{2}^{2} \\
& +2^{-1} \tau^{2} \int_{G} d g\left(\widetilde{M}_{n} * M_{n}\right)(g)\left\|\partial_{g} f\right\|_{2}^{2} .
\end{aligned}
$$

On the right side, the first term is estimated below by $c^{-1} \Gamma_{2}(f)^{2}$, while from (1) and (11) one easily sees that the remaining terms have absolute value less than $c^{\prime}|\tau| \Gamma_{2}(f)^{2}$ for all $|\tau| \leq 1$. Hence we may choose $s_{0} \in(0,1)$ small enough that

$$
\|f\|_{2}^{2}-\left\|K_{n}^{\tau} * f\right\|_{2}^{2} \geq\left(c^{\prime \prime}\right)^{-1} \Gamma_{2}(f)^{2} \quad \text { for all }|\tau|<s_{0} \text { and } n \in \mathbb{N} .
$$

Theorem 3.3 then follows from Theorem 2.8.

EXAmple $(\mathrm{v})$. Let $\left(K_{n}\right)_{n \in \mathbb{N}}$ be symmetric densities satisfying the same assumptions as in Example (i). Let $\left(M_{n}\right)_{n \in \mathbb{N}} \subseteq L^{1} \cap L^{\infty}$ be symmetric real-valued functions with $\int_{G} M_{n}=0$ and satisfying an estimate $\left|M_{n}(g)\right| \leq$ $c e^{-b \varrho(g)^{2}}$ for all $n \in \mathbb{N}$ and $g \in G$. Setting $K_{n}^{\tau}=K_{n}+\tau M_{n}$ for $\tau \in \mathbb{R}$, we have

THEOREM 3.4. There is an $s_{0}>0$ such that an estimate

$$
\left|\left(K_{m+n}^{\tau_{n}} * \cdots * K_{m+1}^{\tau_{1}}\right)(g)\right| \leq c^{\prime} n^{-D / 2} e^{-b^{\prime} \varrho(g)^{2} / n}
$$

holds for all $m \in \mathbb{N}_{0}, n \in \mathbb{N}$ and $\tau_{1}, \ldots, \tau_{n} \in \mathbb{R}$ satisfying $\left|\tau_{j}\right|<s_{0}$ for all $j$.

Proof. Let $Q_{n, \lambda}^{\tau}, \lambda \in \mathbb{R}$, denote the quadratic forms associated with $K_{n}^{\tau}$. According to the proof of Theorem 3.3, for some $s_{0} \in(0,1)$ we have $Q_{n, 0}^{\tau}(f) \geq c^{-1} \Gamma_{2}(f)^{2}$ for all $|\tau|<s_{0}$ and $n \in \mathbb{N}$. Moreover, using $\int_{G} K_{n}^{\tau}=1$ and symmetry of $K_{n}^{\tau}$, one may show in the same way as in Example (i) that

$$
\left|Q_{n, \lambda}^{\tau}(f)-Q_{n, 0}^{\tau}(f)\right| \leq \varepsilon \Gamma_{2}(f)^{2}+c(\varepsilon) \lambda^{2}\|f\|_{2}^{2}
$$


uniformly for all $|\tau| \leq 1, \varepsilon>0, n \in \mathbb{N}$ and $|\lambda| \leq 1$. Then, by the proof of Corollary 2.7, the assumptions of Theorem 2.1 hold uniformly for $|\tau|<s_{0}$, so that it suffices to apply Theorem 2.1.

Acknowledgements. This work was carried out with financial support from the Australian Research Council (ARC) Centre of Excellence for Mathematics and Statistics of Complex Systems (MASCOS).

\section{References}

[1] G. Alexopoulos, Centered densities on Lie groups of polynomial volume growth, Probab. Theory Related Fields 124 (2002), 112-150.

[2] -, Random walks on discrete groups of polynomial volume growth, Ann. Probab. 30 (2002), 723-801.

[3] T. K. Carne, A transmutation formula for Markov chains, Bull. Sci. Math. 109 (1985), 399-405.

[4] N. Dungey, On Gaussian kernel estimates on groups, Colloq. Math. 100 (2004), 77-90.

[5] - Some conditions for decay of convolution powers and heat kernels on groups, Canad. J. Math. 57 (2005), 1193-1214.

[6] - Properties of random walks on discrete groups: time regularity and off-diagonal estimates, preprint, Univ. of New South Wales, 2005.

[7] A. F. M. ter Elst and D. W. Robinson, Gaussian bounds for complex subelliptic operators on Lie groups of polynomial growth, Bull. Austral. Math. Soc. 67 (2003), 201-218.

[8] W. Hebisch and L. Saloff-Coste, Gaussian estimates for Markov chains and random walks on groups, Ann. Probab. 21 (1993), 673-709.

[9] E. Hewitt and K. A. Ross, Abstract Harmonic Analysis I, 2nd ed., Grundlehren Math. Wiss. 132, Springer, New York, 1979.

[10] N. T. Varopoulos, Long range estimates for Markov chains, Bull. Sci. Math. 109 (1985), 225-252.

[11] - Geometric and potential theoretic results on Lie groups, Canad. J. Math. 52 (2000), 412-437.

[12] N. T. Varopoulos, L. Saloff-Coste and T. Coulhon, Analysis and Geometry on Groups, Cambridge Tracts in Math. 100, Cambridge Univ. Press, Cambridge, 1992.

School of Mathematics

The University of New South Wales

Sydney 2052, Australia

E-mail: n.dungey@unsw.edu.au

Received July 25, 2005

Revised version September 27, 2006 